\title{
Los estados inmaduros de Coelosis biloba (Coleoptera: Melolonthidae: Dynastinae) y notas sobre su biología
}

\author{
Immature stages of Coelosis biloba (Coleoptera: Melolonthidae: Dynastinae) with notes on \\ their biology
}

\author{
Luis Carlos Pardo-Locarno', Miguel Ángel Morón ${ }^{2 *}$ y Andreas Gaigl ${ }^{3}$ \\ ${ }^{1}$ Programa Manejo Integrado de Plagas, Centro Internacional de Agricultura Tropical (CIAT) Apartado Aéreo 6713, Cali, Colombia. Dirección \\ actual: Vegetales Orgánicos, CTA, Cali, Colombia. \\ ${ }^{2}$ Departamento de Entomología, Instituto de Ecología, A.C. Apartado Postal 63, Xalapa, Veracruz 91000, México. \\ ${ }^{3}$ Programa Manejo Integrado de Plagas, Centro Internacional de Agricultura Tropical (CIAT) Apartado Aéreo 6713, Cali, Colombia. \\ *Correspondencia: moron_ma@ecologia.edu.mx
}

\begin{abstract}
Resumen. Se describen la larva de tercer estadio y las pupas de macho y hembra de Coelosis biloba (Linné 1767) con ejemplares recolectados en Colombia (Cauca y Valle) y en México (Veracruz) asociados con los hormigueros de Atta cephalotes (Linné 1750). Se incluyen ilustraciones de las estructuras diagnósticas, una clave para separar las larvas de tercer estadio hasta ahora conocidas de la tribu Oryctini en América, y observaciones sobre la biología de C. biloba como inquilino de los nidos de hormigas.
\end{abstract}

Palabras clave: Scarabaeoidea, larva, pupa, taxonomía, mirmecofilia, Colombia, México.

\begin{abstract}
The larva of third instar, male and female pupae of Coelosis biloba obtained inside ant nests of Atta cephalotes in Colombia (Cauca, Valle) and Mexico (Veracruz) are described. Drawings of diagnostic structures, a key to the known third instar larvae of American Oryctini, and observations on the biology of C. biloba as inquiline of ant nests, are included.
\end{abstract}

Key words: Scarabaeoidea, larva, pupa, taxonomy, myrmecophilia, Colombia, Mexico.

\section{Introducción}

De acuerdo con Endrödi (1985) el género Coelosis Hope 1837 está formado por 7 especies, C. bicornis (Leske 1879), C. biloba (Linné 1767), C. bourgini Dechambre 1976, C. denticornis Arrow 1937, C. hippocrates (Blanchard 1846), C. inermis (Sternberg 1908), y C. sylvanus (Fabricius 1775), todas restringidas a Sudamérica, excepto C. biloba que se ha registrado desde el norte de Argentina hasta el sureste de México. A pesar de ser una especie relativamente común, poco se conoce de su biología. Los aspectos biológicos preliminares sobre $C$. biloba fueron descritos por Eidmann (1937), quien señaló por primera vez la asociación entre los estados inmaduros de este escarabajo y los nidos de hormigas Atta sexdens (Linné 1758) en Brasil, comentando que los huevos del dinastino son colocados en las cámaras subterráneas

Recibido: 29 junio 2005; aceptado: 22 mayo 2006 donde las hormigas preparan las hojas cortadas antes de llevarlas a las cámaras donde se cultivan los hongos con los cuales se alimenta la colonia. Según Eidmann (1937), las hormigas llevan los huevos y las larvas pequeñas a las cámaras donde se cultivan los hongos, y allí se desarrollan sin ser molestadas por sus anfitrionas. Lachaume (1992) planteó la posibilidad de que C. biloba simplemente fuera una especie con hábitos mirmecófilos. Morón et al. (1997) proporcionaron un compendio bioecológico de C. biloba en México, indicando que: "habita bosques tropicales perennifolios y caducifolios, comunidades vegetales secundarias y plantaciones tropicales establecidas entre el nivel del mar y los $1500 \mathrm{~m}$ de altitud; los adultos tienen hábitos nocturnos y son atraídos por las luces eléctricas entre mayo y noviembre pero se desconocen sus hábitos de alimentación. Las larvas y adultos tenerales se han encontrado dentro del suelo cerca de las cámaras donde las hormigas del género Atta cultivan los hongos que les sirven de alimento". Navarrete-Heredia (2001) registró la 
asociación de este escarabajo con nidos de hormigas Atta cephalotes (Linné 1750) y A. mexicana (Smith 1858) en la región neotropical, y Ratcliffe (2003) comentó sobre la asociación de adultos con nidos de hormigas Atta en Honduras, agregando que la distribución altitudinal de esta especie en Panamá y Costa Rica abarca desde el nivel del mar hasta los $1200 \mathrm{~m}$ de altitud. Ninguno de estos autores hizo referencia a la morfología larvaria, ni describió los estados inmaduros. Esta publicación tiene como objetivos describir la larva de tercer estadio y las pupas de macho y hembra de C. biloba, y aportar observaciones sobre su biología y hábitos mirmecófilos.

\section{Materiales y métodos}

En el transcurso del trabajo de campo realizado por el primer autor (LCPL) en Colombia se exploraron varios nidos de hormigas Atta durante agosto a noviembre de 2002 en Palmira, corregimiento de Calucé, localidad Alto del Tigre, a 1623-1685 m (N 03 32' 779'“ y O $\left.76^{\circ} 10^{\prime} 748^{\prime \prime}\right)$; y desde febrero a mayo de 2003 en Valle del Cauca, Caldono, vereda La Campiña, 1450 m snm. Los hormigueros seleccionados fueron excavados hasta 1.5-2.0 $\mathrm{m}$ de profundidad, los adultos de C. biloba presentes en el suelo se identificaron con facilidad, pero las larvas requirieron de cría en cautiverio hasta obtener una identificación positiva. Las larvas más grandes que se encontraron en ese ambiente se guardaron en recipientes plásticos transparentes, con capacidad de 20-24 litros, mantenidos a $21-25^{\circ} \mathrm{C}$, cuyos costados fueron cubiertos con plástico negro, y rotulados con los datos de localidad y fecha de colecta. El sustrato puesto en estos recipientes fue tierra del hormiguero con el contenido de una cámara fúngica. Cada 15 días se examinaron los terrarios hasta verificar la construcción de cámaras pupales y la aparición de adultos. Después de identificar a los primeros adultos, varias larvas, pupas de macho y de hembra, incluidas sus exuvias, fueron fijadas en agua hirviendo por 2 minutos, para conservarlas en solución de formol al 10\%. Las recolectas en los hormigueros de Colombia permitieron reunir 28 larvas de tercer estadio, 10 pupas y 7 adultos de esta especie. A ellos adicionamos una larva de tercer estadio y un adulto recolectados en un hormiguero de Atta cephalotes explorado en Córdoba, Veracruz, México. Las piezas bucales fueron disectadas para la descripción, y con la ayuda de un estereomicroscopio provisto de cámara clara y cámara fotográfica, se realizaron las ilustraciones de las estructuras diagnósticas que apoyan las descripciones. La terminología empleada es la propuesta por Ritcher (1966) y Morón (1987). Los ejemplares estudiados se conservan en las colecciones Familia Pardo-Locarno (CFPL) e Instituto de Ecología, A.C. Xalapa, Veracruz (IEXA).

\section{Descripción}

Coelosis biloba (Linné 1767) (Figs. 1-20)

Larva de tercer estadio. Aspecto general (Fig. 1). Longitud total aproximada por el dorso del cuerpo: 79.1- 85.1 mm; cabeza parda obscura, pequeña en proporción al cuerpo, con las antenas, las mandíbulas y el ápice de los palpos maxilares negros. Cuerpo blanco cremoso, casi cilíndrico, regiones dorsal y ventral casi glabras, regiones laterales con un número moderado de sedas concentradas en las áreas pedales; escleromas protorácicos pardo rojizos; patas con los dos artejos basales amarillos y los dos artejos distales pardo obscuros, casi negros.

Cabeza (Fig. 2). Anchura máxima de la cápsula cefálica: $7.58 \mathrm{~mm}$. Superficie del cranium punteada rugosa,

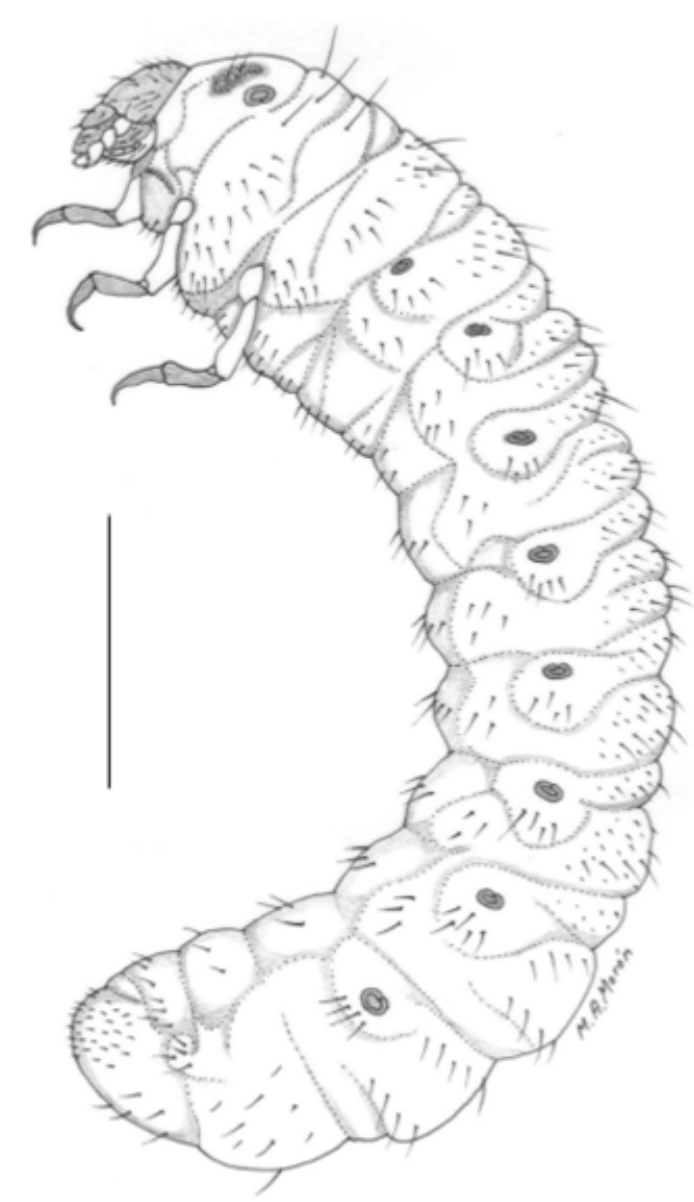

Figura 1. Coelosis biloba (Linné) larva de tercer estadio al inicio de la etapa de prepupa. Línea de escala equivalente a $2 \mathrm{~cm}$. 

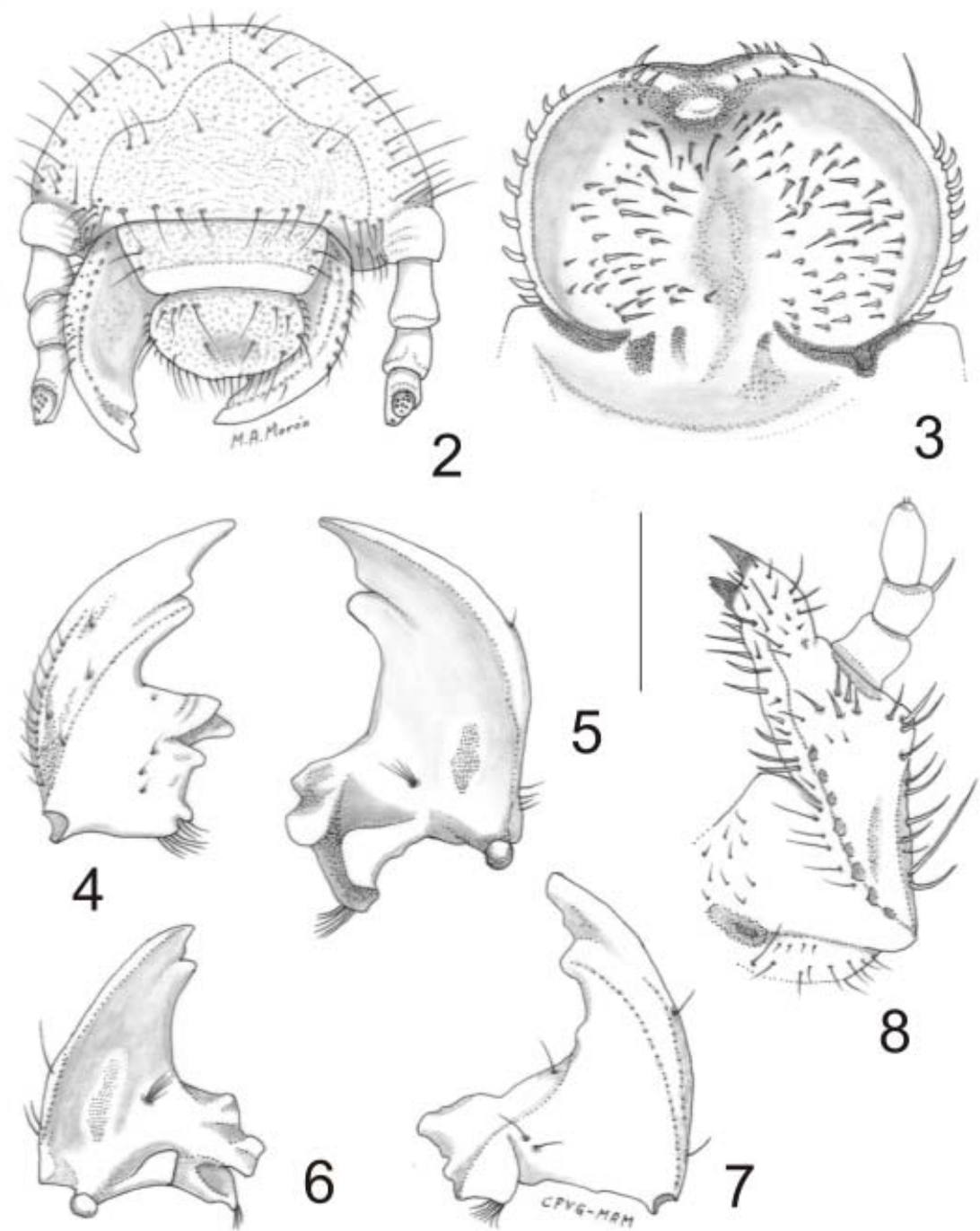

Figuras 2-8. Coelosis biloba, larva de tercer estadio: 2. Aspecto frontal de la cápsula cefálica. 3. Epifaringe. 4. Vista dorsal de la mandíbula izquierda. 5. Vista ventral de la mandíbula izquierda. 6. Vista ventral de la mandíbula derecha. 7. Vista dorsal de la mandíbula derecha. 8. Vista dorsal de la maxila derecha. Líneas de escala equivalentes a $1 \mathrm{~mm}$.

ligeramente brillante. Frente con dos o tres fosetas rugosas alargadas muy someras en la región central, una seda frontal posterior y 2 sedas frontales exteriores a cada lado, con 6-10 sedas frontales anteriores largas y 5-8 sedas en cada ángulo anterior de la frente; el resto de la superficie craneal con 25 sedas dorso-epicraneales, 2-5 sedas epicraneales largas y 4-6 sedas basi-antenales a cada lado. Clípeo punteado rugoso, con 2 sedas a cada lado y 4 sedas alineadas a lo largo del límite del preclypeus y el postclypeus, o sin ellas. Labro asimétrico, ligeramente bilobulado, intensamente rugoso-punteado, con fosetas poco profundas, una hilera irregular de 6-8 sedas ubicada entre los ángulos posteriores y la región central, un par de sedas gruesas centrales y 8-12 sedas cerca del borde anterior. Ocelli vagamente marcados por manchas amarillentas, con forma irregular. Epifaringe (Fig. 3) con epizygum poco definido y zygum en forma de proceso haptomeral, protuberante, pigmentado, en posición oblicua, de forma piramidal, rodeado por un grupo de 6-8 sedas robustas. Acroparia conformada por grupos de 6-7 sedas robustas a cada lado, dirigidas hacia el ápice y un grupo central de 5-6 sedas robustas. Chaetoparia con escasas sedas gruesas en torno del pedium y sensilas dispersas bordeando las gymnoparia. Chaetoparia izquierda con 28-30 sedas gruesas y 18-22 sensilas. Chaetoparia derecha con $38-42$ sedas gruesas y 20-22 sensilas. Gymnoparia amplias, más ensanchadas 
en su mitad distal. Acanthoparia formada por 8-14 sedas cortas con forma de hoja de navaja. Laeotorma estrecha con la pternotorma corta, redondeada, protuberante y pigmentada, epitorma fina. Dexiotorma engrosada en su porción externa y afilada en el extremo proximal, apenas alcanza la nesia externa. Nesia externa o placa esclerosada con perfil triangular, pigmentada, dentiforme y prominente, con un grupo de 3-4 sedas pequeñas en su esquina distal. Nesia interna o cono sensorial poco protuberante $y$ suavemente pigmentado. Crepis dividido, poco notable, ligeramente pigmentado en su borde izquierdo. Pedium con áreas pigmentadas de diferentes tamaños. Mandíbula izquierda (Figs. 4 y 5) con el ápice agudo y la escotadura incisiva profundamente marcada entre los dentículos segundo y tercero; borde postincisivo inerme; región molar formada por tres lóbulos bien definidos; acia pequeña y brustia formada por 8-10 sedas medianas amarillas. Scrobis con un surco amplio que inicia cerca del preartis, se ensancha llegando al tercio distal, donde culmina en una foseta, con una seda robusta. Superficie ventral con el área estriduladora de perímetro ahusado, formada por 28-32 surcos transversales finos, y con 6-7 sedas gruesas y largas situadas en un punto profundo cercano a la base de la región molar. Mandíbula derecha (Figs. 6 y 7) con el ápice agudo y la escotadura incisiva amplia; borde postincisivo con un pequeño dentículo poco prominente; región molar amplia, ensanchada, formada por dos o tres lóbulos mal definidos. Calix semitriangular, proyectado hacia la base; brustia formada por 8-10 sedas robustas y largas. Superficie ventral con el área estriduladora más estrecha que la de la mandíbula izquierda, formada por 3035 surcos transversales finos, y con 6-7 sedas gruesas y largas situadas en un punto profundo cercano a la base de la región molar. Scrobis similar a la mandíbula izquierda. Maxila (Fig. 8) con el uncus de la galea notablemente largo, y la lacinia con dos unci apicales largos poco separados y un diente pequeño poco notable situado en la parte proximal de su base. Área estriduladora maxilar compuesta de una hilera irregular de 6-7 dientecillos cortos de base ancha y ápice agudo o redondeado, precedidos por una proceso truncado pigmentado. Artejos de los palpos maxilares cortos, ensanchados y redondeados. Labium (Fig. 9) con una hilera de 7-8 sedas delgadas laterales, 6 sedas medianas ubicadas entre las bases de los palpos, y 60-70 sedas robustas ubicadas sobre la glosa; escleroma hipofaríngeo asimétrico, pigmentado, con un proceso dentiforme aguzado y notablemente elevado en el lado derecho; lóbulos hipofaríngeos con un grupo de 4-5 sedas en cada borde lateral. Artejos de los palpos labiales ligeramente alargados. Antenas proporcionalmente cortas y robustas (Fig. 2), con el primer artejo más largo que cualquiera de los otros, con el ápice dilatado, el segundo artejo de igual forma pero más corto que el precedente, el tercero con una proyección ventral que alcanza la mitad de la longitud del artejo apical, el cual es muy corto, piriforme (Fig. 10). El artejo apical presenta 8-11 áreas sensoriales dorsales de diferentes tamaños, 13-19 áreas sensoriales ventrales, y 3-4 sensores filiformes apicales (Figs. 11-12). El ápice de la proyección ventral del tercer artejo antenal tiene un área sensorial ovalada.

Tórax (Fig. 1). Protórax con escleromas laterales amplios de perímetro irregular, provistos con 2-4 sedas largas y erectas. Estigma respiratorio protorácico con $1.95 \mathrm{~mm}$ de altura y $1.48 \mathrm{~mm}$ de anchura; lóbulos de la placa respiratoria contiguos y dirigidos hacia la parte posterior; bulla plana, con un surco transversal, irregular. Pronoto con dos hileras transversales irregulares de 10-12 sedas medianas a largas; mesonoto y metanoto con hileras alternadas de 10-16 sedas medianas a largas, muy irregulares, intercaladas con escasas sedas espiciformes muy cortas. Las áreas pedales concentran grupos de 18-22 sedas largas. Las patas protorácicas son ligeramente más cortas que las patas mesotorácicas, y éstas son ligeramente más cortas que las metatorácicas. En todas el artejo tarsal es más largo que la tibia (Fig. 13). Los tarsúngulos (Figs. 14-15) son muy largos, con el ápice aguzado y recurvado, con una seda larga y delgada en los lados anterior y posterior de su base.

Abdomen. La región dorsal de cada uno de los segmentos I a VII tiene entre 56 y 60 sedas organizadas en dos hileras transversales irregulares; la mayor parte de estas sedas son muy cortas, espiciformes, y una minoría son sedas delgadas y largas. En la región dorsal del segmento VIII existen dos hileras transversales con 28 a 30 sedas medianas o largas cada una, claramente separadas. Las regiones dorsales de los segmentos IX y $\mathrm{X}$ sólo presentan algunas sedas medianas muy esparcidas. Los estigmas respiratorios abdominales (Fig. 16) incrementan gradualmente su tamaño del primero al octavo segmento, desde $1.3 \mathrm{~mm}$ de altura y $1.0 \mathrm{~mm}$ de anchura hasta $1.8 \mathrm{~mm}$ de altura y 1.4 $\mathrm{mm}$ de anchura. Raster sin palidia ni septula; teges formado por 70-80 sedas robustas y cortas, barbula representada por 4-6 sedas medianas o largas; labio anal superior con 70-80 sedas muy cortas y robustas.

Material examinado: 2 larvas colectadas en COLOMBIA: Valle, Palmira, corregimiento Calucé, Alto del Tigre, fijadas 21-XII- 2002, en nido de Atta, 1,660 m, R. Zúñiga, J. C. Noguera y L. C. Pardo-Locarno (CFPL). Una larva y 2 exuvias de larva de tercer estadio procedentes de COLOMBIA: Cauca, Caldoso, vereda La Campiña, fijadas 12-VI-2003, en nido de Atta, 1450 m, H. Trujillo (CFPL). Una larva colectada en MÉXICO: Veracruz, Córdoba, Tlacotengo, fijada 28-II-1984, en nido de Atta, 806 m, I. Landero (IEXA-XX-EC-184). 

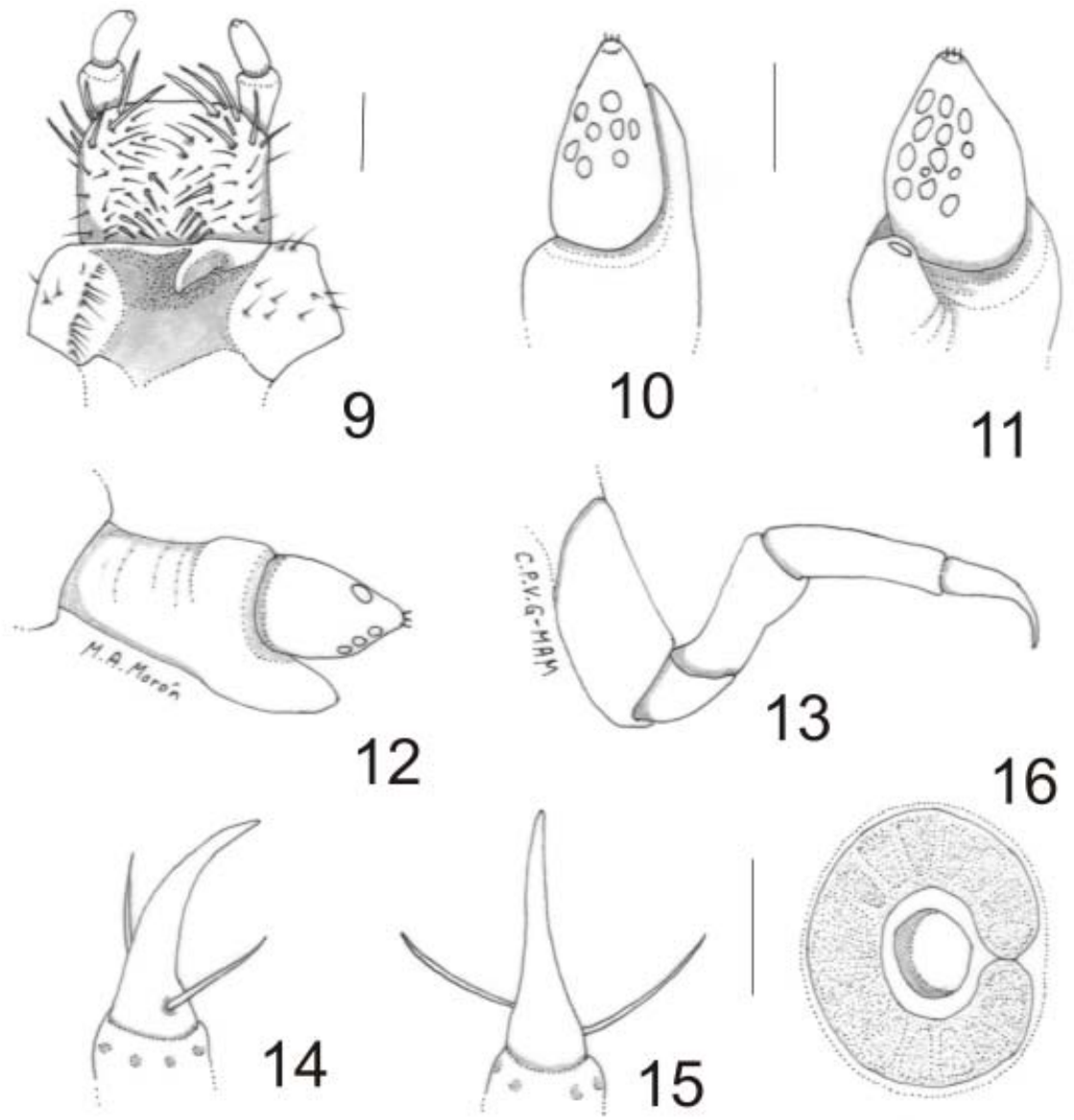

Figuras 9-16. Coelosis biloba, larva de tercer estadio: 9. Labio hipofaringe. 10. Vista lateral de los artejos apicales de la antena. 11. Vista dorsal del último artejo antenal. 12. Vista ventral del último artejo antenal. 13. Vista postero-lateral de una pata metatorácica. 14. Vista posterior del meso-tarsúngulo derecho. 15. Vista lateral del meta-tarsúngulo derecho. 16. Aspecto lateral de la placa respiratoria derecha del octavo segmento abdominal. Líneas de escala equivalentes a $0.5 \mathrm{~mm}$, excepto en las figs. 9 y $13=1 \mathrm{~mm}$.

Pupas. Macho. Aspecto general (Figs. 17-18). Longitud 55-58 mm, anchura máxima 24-26 mm. Exarata. Cuerpo robusto, de forma oval alargada, amarillo pardusco, con lustre moderado; en su mayor parte glabro, a excepción de una fina cubierta de microtrichia dorada en la mitad distal del abdomen.

Cabeza. Dirigida ventralmente, en forma oblicua al plano del cuerpo, con antenas, palpos, labium y borde anterior del clípeo bien definidos; ojos compuestos poco visibles, cubiertos por el ángulo pronotal anterior; borde del clípeo redondeado; en el dorso de la región frontoclipeal de los machos teloceros (Fig. 18) predomina un cuerno muy robusto, cuya base cubre desde el borde occipital hasta cerca al ápice clipeal; dicho cuerno cefálico inicialmente se proyecta perpendicularmente al plano de la cabeza, y en su parte media se dobla en ángulo recto hacia arriba. Ápice del clípeo redondeado.
Tórax. Pronoto más ancho que largo y en vista lateral más grueso hacia su base; en el disco pronotal de los machos teloceros (Fig. 17) predominan dos proyecciones divergentes grandes, anchas, deprimidas, con los ápices redondeados; el borde posterior del pronoto casi recto, y los ángulos anteriores proyectados en ángulo obtuso hacia el frente y hacia abajo, de modo que oculta gran parte de los ojos y bordea la base del cuerno cefálico.

Dorso del meso y metatórax con la parte central ampliamente convexa. Las pterotecas se curvan hacia los lados y la región ventral sobre el segundo y tercer par de podotecas, el ápice de los pterotecas alcanza los extremos apicales del tercer par de podotecas. Patas: protibia con tres proyecciones sobre el borde externo; mesotibia con dos proyecciones sobre el borde externo y dos angulosidades en el ápice; metatibia con una muesca en el cuarto distal del borde externo y dos angulosidades en el ápice interno. 

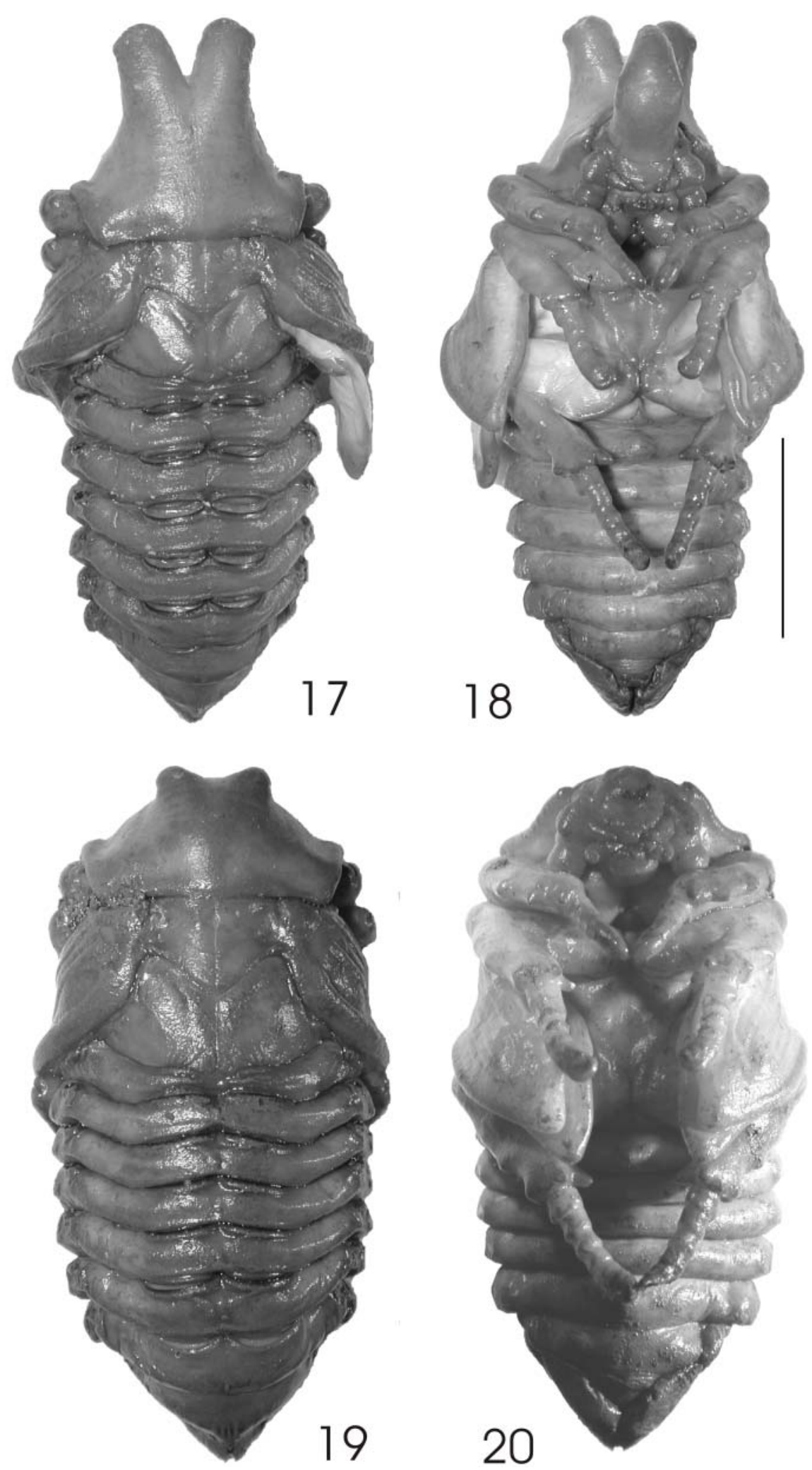

Figuras 17-20. Coelosis biloba, pupas: 17. Aspecto dorsal de un macho telocero. 18. Aspecto ventral de un macho telocero. 19. Aspecto dorsal de una hembra. 20. Aspecto ventral de una hembra. Línea de escala equivalente a $2 \mathrm{~mm}$. 
Clave para separar las larvas de tercer estadio de los géneros americanos de Dynastinae, Oryctini (basada en Ritcher, 1966; Morón, 1987; Morón y Ratcliffe, 1990; Álvarez-Castillo et al., 1998; Morelli y Morón, 2003; Ratcliffe, 2003).

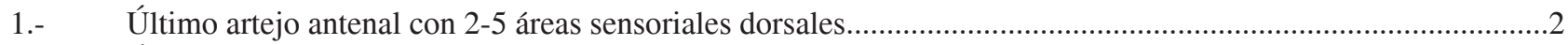

1' Último artejo antennal con 6 o más áreas sensoriales dorsales...................................................................

2.- $\quad$ Raster con septula ancha, extendida sobre el labio anal inferior; palidia polístico, cada palidium formado por 2-3 hileras irregulares de pali espiniformes. Ocelos ausentes. Último artejo antenal con 5 áreas sensoriales dorsales. Heterogomphus (s.str.) Burmeister 1847 Raster sin septula ni palidia. Ocelos bien definidos. Último artejo antenal con 2-5 áreas sensoriales dorsales Xyloryctes Hope 1837

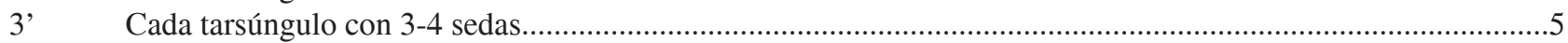

4.- $\quad$ Artejos antenales largos, el segundo cuando menos dos veces más largo que ancho, y el cuarto casi tan largo como el tercero. La longitud de cada tarsúngulo no alcanza la mitad de la longitud del artejo precedente. Placas respiratorias de los segmentos abdominales I a VIII del mismo tamaño. Enema Hope 1837 Artejos antenales cortos y anchos, el segundo tan largo como ancho, y el cuarto con la mitad de la longitud del tercero (Fig. 10). La longitud de cada tarsúngulo es mayor que la mitad de la longitud del artejo precedente. El tamaño de las placas respiratorias de los segmentos abdominales se incrementa progresivamente del I al VIII. Coelosis Hope 1837 Cabeza sin sedas frontales anteriores. Placas respiratorias de segmentos abdominales I a V más grandes que las placas de los segmentos VI a VIII. Anchura máxima de la cápsula cefálica 14-15 mm.

..Heterogomphus (Dineterogomphus) Prell 1912

Cabeza con 2-8 sedas frontales anteriores. Placas respiratorias de los segmentos abdominales I a VIII casi del mismo tamaño (excepto en Strategus splendens (Beauvois) donde la placa del segmento VIII es mucho más pequeña que las precedentes). Anchura máxima de la cápsula cefálica 9-12 mm..... Strategus Hope 1837

Tarsómeros delineados, progresivamente alargados del primero al tercer tarsómero, el cual es el más largo de la región tarsal.

Abdomen. Con nueve segmentos visibles, estigmas respiratorios del segmento I parcialmente ocultos por la pterotecas, estigmas respiratorios de los segmentos II, III y IV con peritrema anular, esclerosado y pigmentado, prominentes, dirigidos hacia delante; estigma respiratorio del segmento $\mathrm{V}$ ocluido, poco prominente, en forma de roseta; estigmas respiratorios de los segmentos VI y VII ocluidos y deformados como grandes rugosidades irregularmente trazadas. Área tergal de los segmentos I - VI con seis pares de órganos dioneiformes prominentes y pigmentados (Fig. 17), cada uno con $4 \mathrm{~mm}$ de anchura y 1,5 mm de apertura; el último par de órganos dioneiformes con los bordes poco pigmentados. Terguito VIII más angosto, con las regiones laterales ligeramente excavadas. Último terguito semitriangular, con el ápice terminado en dos prominencias angulosas breves, cubiertas con sedas doradas, muy cortas. Región ventral del último segmento (Fig. 18) conformada por dos estructuras semitriangulares prominentes, contiguas, convergentes, separadas por un surco apical, en medio se encuentra el ámpula genital, convexa, con una proyección ancha, corta, cuyo ápice es trilobulado, el cilindro central apicalmente surcado, flanqueado por depresiones semitriangulares.

Hembra. Aspecto general (Figs. 19-20). Forma y coloración similar al macho. Longitud 33-39 mm, anchura máxima 23-24 mm. Cabeza con la región frontoclipeal convexa y redondeada; disco del pronoto dominado por dos proyecciones robustas, cortas, divergentes, con ápices redondeados (Fig. 19); ámpula genital cóncava, amplia, con rugosidades transversales y dos pequeños tubérculos semiesféricos contiguos, apenas visibles (Fig. 20).

Material examinado: 2 pupas (macho y hembra) colectadas en campo y 5 pupas ( 3 machos, 2 hembras) obtenidas de la cría de larvas de tercer estadio colectadas en COLOMBIA: Valle, Palmira, corregimiento Calucé, Alto del Tigre, en nido de Atta, 1680 m, fijadas en noviembre 2002, J. C. Noguera y L. C. Pardo-Locarno (CFPL). Tres pupas (2 machos, 1 hembra) colectadas en COLOMBIA: Cauca, Caldoso, Vereda La Campiña, en nidos de Atta, 1450 m, fijadas en enero 2003, H. Trujillo (CFPL).

Comentarios taxonómicos. En el campo la larva de C. biloba se distingue fácilmente de otros Dynastinae Oryctini por su asociación con los nidos de hormigas Atta, siendo la larva más grande de todas las observadas en 
tal circunstancia. Su morfología combina un cuerpo con poca vestidura setífera, cabeza y patas muy pigmentadas, artejos antenales cortos y robustos, el tercero de ellos con una proyección ventral grande y el artejo apical muy corto; las patas tienen el tarsúngulo muy largo, recurvado y con 2 sedas prebasales. La pupa del macho presenta los rasgos distintivos de la cornamenta cefálica y torácica observada en el adulto, mientras que la pupa de la hembra presenta prominencias en el pronoto, las cuales desaparecen en la hembra adulta.

Observaciones sobre la biología y distribución de $C$. biloba. En varias ocasiones se habían recolectado adultos muertos o pupas en cámaras excavadas en la tierra de los hormigueros de especies de Atta (por ejemplo, en 1984 en El Carmen, Valle, Colombia, a $1500 \mathrm{~m}$ snm, y en 1985 en Timbío, Cauca, Colombia, a 1450 m snm), sin embargo, sólo durante los trabajos realizados por PardoLocarno y colaboradores durante 2002 y 2003 los nidos fueron excavados profundamente encontrando larvas desarrolladas, prepupas y pupas habitando en la tierra en torno a las cámaras y galerías de Atta cephalotes, en donde la hormiga cultiva el hongo simbiótico Rozites gongylophora Moeller 1893 (Basidiomycetes). A pesar de varias excavaciones exitosas, no fue posible observar larvas o inmaduros en el interior de las cámaras de hongos o en contacto directo con hormigas; al contrario, se observó que las hormigas atacaron a las larvas cuando éstas eran descubiertas en las excavaciones, aunque esto puede ser explicado por el disturbio que implica la destrucción del nido. Una situación parecida se registró durante febrero de 1984 en Tlacotengo, Fortín de Las Flores, Veracruz, México (Landero, 1985).

Las larvas de tercer estadio mantenidas en cautiverio demoraron de seis a ocho semanas en pasar a prepupa, las cuales luego de tres semanas en la cámara pupal construida con tierra compactada, mudaron a pupa, estado en el cual demoraron de tres a cuatro semanas. Los adultos tenerales permanecieron hasta dos semanas dentro de la cámara, antes de excavar túneles para refugiarse durante el día, ya que sólo se les observó activos en el exterior durante la noche.

Variosadultos fueron recolectadosdurantelaexcavación de nidos, en el suelo removido por la construcción de galerías, otros se observaron superficialmente enterrados, pero no se observaron adultos del escarabajo en contacto directo con los corredores de las hormigas, cerca de cámaras fúngicas o cúmulos de detritus. Los adultos de los dos sexos son atraídos por las luces eléctricas de tipo incandescente, fluorescente o de vapor de mercurio.

Algunos adultos obtenidos de la cría de larvas o, capturados directamente en el campo, se mantuvieron en cautiverio durante semanas, pero no se alimentaron de los trozos de frutas que se les ofrecieron. Otras especies de Dynastinae observadas en cautiverio, como Strategus aloeus (Linné 1758), Enema pan (Fabricius 1775), Dynastes hyllus Chevrolat 1843 o Megasoma elephas (Fabricius 1775), si se alimentan con trozos de frutos de naranja, plátano, zapote, o mango. Sin embargo, algunos adultos de C. biloba obtenidos en la cría de laboratorio destrozaron larvas de su misma especie, y aparentemente consumieron sus tejidos blandos. Tal fenómeno de canibalismo puede derivarse de las condiciones de cautiverio, aunque existen antecedentes de ello en otras especies de Dynastinae Phileurini como Phileurus valgus (Olivier 1789) y $P$. didymus (Linné 1758), P. truncatus (Beauvois 1807) y Hemiphileurus dejeani (Bates 1889) que han sido observados en cautiverio consumiendo larvas y adultos de otros coleópteros, incluyendo dinastinos grandes como Heterogomphus chevrolati Burmeister 1847 (Morón et al., 1997). En la localidad de Caldono, Cauca, Colombia, fue posible recolectar larvas de C. biloba afectadas por avispas parasitoides, cuyos adultos se obtuvieron en cautiverio y correspondieron a una avispa grande de una especie no identificada del género Campsomeris (Hymenoptera: Scoliidae).

En el catálogo de Coleoptera Melolonthidae de Colombia, Restrepo et al. (2003) registraron a C. biloba en los departamentos de Antioquia, Risaralda, Tolima y Valle, pero en una revisión reciente de las colecciones colombianas, sobre todo del primer autor (LCPL) se encontraron 103 ejemplares recolectados en las siguientes localidades: Antioquia (Bello, Cocorná, Fredonia, Puerto Pinzón, Apartadó), Boyacá (Otanche), Cundinamarca (El Colegio, Sasaima, Viotá), Cauca (Caldono, Popayán, Santander de Quilichao), César (San Alberto), Chocó (Togoromá), Córdoba (Monteria, Pueblo Nuevo), Huila (Rivera), Magdalena (Santa Marta), Meta (Villavicencio, Cumaral), Nariño (La Unión), Putumayo (Mocoa), Quindio (La Tebaida), Risaralda (La Florida, Santa Rosa), Santander del Sur (Río Minero, La Belleza, La Paz), Tolima (Ibagué, China Alta, La Arabia) y Valle (Buga, Buenaventura, Cali, Cartago, Calima, Dagua, Málaga, Palmira, Pance, Tulúa).

Estos registros amplían notablemente el conocimiento sobre la distribución de esta especie en Colombia y evidencian su capacidad para habitar en formaciones ecológicas muy variadas, que van desde el bosque tropical muy seco hasta las selvas pluviales, abarcando un amplio rango altitudinal que ocupan las selvas bajas y las selvas andinas, desde el nivel del mar hasta los $1680 \mathrm{~m}$ de altitud, coincidente con la amplia distribución de las hormigas del género Atta. En el sureste de México, C. biloba está limitado a los ambientes con bosque tropical caducifolio o perennifolio con influencia del Golfo de México y el 
Mar Caribe situados entre el nivel del mar y los $1500 \mathrm{~m}$ de altitud, y no se le ha registrado en la vertiente del Pacífico o en altitudes superiores, aún cuando las colonias de Atta estén presentes y sean abundantes.

\section{Discusión}

Nuestras observaciones no permiten corroborar lo expuesto por Eidmann (1937), respecto a una asociación estrecha entre los inmaduros de C. biloba y las hormigas. Por ahora, la evidencia obtenida apunta al inquilinismo del escarabajo en la vecindad de los nidos de Atta, en una relación que parece beneficiar al escarabajo y que poco perturba a las hormigas; algo similar a lo observado en algunos géneros de Cetoniinae Gymnetini (por ejemplo, Cotinis; Morón et al., 1997; Pardo-Locarno y Orozco, 2002) cuyas larvas aprovechan el suelo removido, rico en materia orgánica y desperdicios, sin que las larvas entren en contacto con las hormigas. No obstante el planteamiento de una relación de inquilinismo, lo observado confirma que C. biloba tiene hábitos mirmecófilos, bastante diferentes de lo que se conoce en otras especies de Dynastinae Oryctini, cuyas larvas son saprófagas o saproxilófagas (Pardo-Locarno et al., 2000). Sin embargo, se requieren más detalles sobre el ciclo vital y el comportamiento de alimentación y reproducción de los adultos de ésta y otras especies de Coelosis para sustentar con mayor profundidad esta discusión.

\section{Agradecimientos}

El primer autor agradece a Rodrigo Zúñiga (CIAT) y Héctor Trujillo (Caldono) su colaboración en el trabajo de campo; a Andrés Felipe Serna (Unal) y Germán Calberto (CUAO) su asistencia en las actividades de laboratorio. Para la sistematización de datos y la elaboración del documento se contó con la asistencia de Claudia Patricia Vélez García. El profesor James Montoya (Universidad del Valle) examinó una versión preliminar del texto y aportó comentarios al documento final. Guillermo Sotelo aportó información valiosa sobre la biología y ecología de las hormigas Atta. Al Centro Internacional de Agricultura Tropical (CIAT) por las facilidades para realizar el trabajo. Miguel Ángel Morón agradece a Ivonne Landero Torres (Universidad Veracruzana, campus Córdoba) el donativo de los ejemplares de coleópteros mirmecófilos obtenidos durante su trabajo de tesis. Este artículo contiene resultados del proyecto de disertación doctoral de Luis Carlos Pardo Locarno, registrado en la Universidad del Valle, Colombia con el título "Contribución al conocimiento sistemático y biológico de los Scarabaeoidea (Coleoptera) de Colombia". También es una contribución al proyecto "Coleópteros Lamelicornios de América Latina" apoyado por el Departamento de Entomología, Instituto de Ecología, A.C. Xalapa (cuenta 902-08-011).

\section{Literatura citada}

Álvarez-Castillo, H. A., A. M. Vincini, A. N. López, D. M. Carmona y P. L. Manetti. 1998. Descripción de los estados inmaduros de Heterogomphus pauson (Perty, 1830) (Coleoptera: Scarabaeidae: Dynastinae). Elytron 12: 97-103.

Endrödi, S. 1985. The Dynastinae of the world. Akadémiai Kiadó. Budapest. 800 p.

Eidmann, H. 1937. Die Gaste und gastverhalnisse der Blattschneiderameise Atta sexdens L. Zeitschcrift fur Morphologie und Okologie der Tiere 32: 391-462.

Lachaume, G. 1992. The beetles of the world. Dynastidae Americains. Sciences Naturelles, Venette, France. 98 p.

Landero, T. I. 1985. Estructura y composición de un nido de Atta cephalotes (L.) en Tlacotengo, municipio de Fortín, estado de Veracruz, México. Tesis licenciatura. Facultad de Ciencias Biológicas, Universidad Veracruzana, Córdoba, Veracruz, México. 98 pp.

Morelli, E. y M. A. Morón. 2003. Descriptions of Xyloryctes Hope larvae with a key to species based on the third stage larvae (Coleoptera: Scarabaeidae: Dynastinae). The Coleopterists Bulletin 57: 289-295.

Morón, M. A. 1987. Los estados inmaduros de Dynastes hyllus Chevrolat (Coleoptera: Melolonthidae; Dynastinae) con observaciones sobre su biología y el crecimiento alométrico del imago. Folia Entomológica Mexicana 72: 33-74.

Morón, M. A. y B. C. Ratcliffe. 1990. Descriptions of Strategus larvae with a key to species based on the larvae (Coleoptera: Scarabaeidae: Dynastinae). Elytron 4: 53-66.

Morón, M. A., B. C. Ratcliffe y C. Deloya. 1997. Atlas de los escarabajos de México, Coleoptera Lamellicornia, vol I, familia Melolonthidae. Sociedad Mexicana de Entomología, A.C. y Comisión Nacional para el Conocimiento y Uso de la Biodiversidad (CONABIO), Xalapa, Veracruz. 280 p.

Navarrete-Heredia, J. L. 2001. Beetles associated with Atta and Acromyrmex ants (Hymenoptera: Formicidae: Attini) Transactions of the American Entomological Society 127: 381-429.

Pardo-Locarno, L. C., J. C. Neita y F. Yepes. 2000. Nota sobre los escarabajos rinoceronte (Coleoptera: Melolonthidae; Dynastinae) de Colombia con especial 
referencia a la Cuenca Calima-San Juan, Valle. In Resúmenes XXXV Congreso Nacional de Ciencias Biológicas (ACCB), Medellín, p.206.

Pardo-Locarno, L. C. y J. Orozco. 2002. Escarabajos Cetoniinae (Coleoptera: Scarabaeiodea) de Colombia: aporte a una visión de conjunto. In Resúmenes XXIX Congreso de la Sociedad Colombiana de Entomología (SOCOLEN), Monteria, p. 58.

Ratcliffe, B. C. 2003. The Dynastine scarab beetles of
Costa Rica and Panama (Coleoptera: Scarabaeidae: Dynastinae). Bulletin of the University of Nebraska State Museum 16: 1-506.

Restrepo-Giraldo, H., M. A. Morón, F. Vallejo, L. C. Pardo-Locarno y A. López-Ávila. 2003. Catálogo de Coleoptera Melolonthidae (Scarabaeidae Pleurosticti) de Colombia. Folia Entomológica Mexicana 42: 239-263. Ritcher, P. O. 1966. White grubs and their allies. Oregon State University Press, Corvallis. 219 p. 